\title{
La articulación del discurso moral y la estructura valórica en jóvenes acogidos en residencias de protección de derecho: Perfil de sujeto moral
}

\author{
Pablo Pérez Valdés*
}

\begin{abstract}
Resumen
El siguiente artículo está desarrollado en base a la investigación "Discurso moral y estructura valórica en jóvenes acogidos en programas residenciales de protección", desarrollada entre el año 2008 y el primer trimestre del 2009.

Esta investigación tenía como objetivo describir la configuración de la estructura valórica y del discurso moral en jóvenes que han sido acogidos en sistemas residenciales de protección, o fuera del núcleo familiar de origen que hipotéticamente afectaría su configuración valórica. En este sentido, lo que se busca es describir las discrepancias y similitudes en el discurso entre los sujetos jóvenes.

La recolección de datos utilizó la técnica de muestreo por cuotas y abarcó un total de 180 personas.

Palabras clave: Jóvenes, discursos, estructura valórica, Programa Residencial de Protección.
\end{abstract}

\begin{abstract}
The article below has been developed based on the research of "Moral discourse and value structure of young people taken in at juvenile court schools", developed in the year 2008 and the first quarter of 2009.

This study aimed to describe the configuration of the value structure, and moral discourse of young people who have been taken in at juvenile court school protection systems, or outside the family home which hypothetically might affect their sense of value. In this respect, it is intended to describe the differences and similarities in the discourse among youngsters.

The data collection used the quota sampling technique and covered a total of 180 people.
\end{abstract}

Key words: youngsters, discourses, value structure, juvenile court school protection programs.

* Sociólogo, Universidad Católica Silva Henríquez, Magíster (c) en Sociología de la Modernización, Universidad de Chile. Encargado Nacional de Desarrollo de Programas, Aldeas Infantiles SOS Chile. pperez@aldeasinfantiles.cl 
La articulación del discurso moral y la estructura valórica en jóvenes acogidos en residencias de protección de derecho: Perfil de sujeto moral

\section{Introducción}

El siguiente artículo está desarrollado en base a la investigación "Discurso moral y estructura valórica en jóvenes acogidos en programas residenciales de protección", desarrollada ente el año 2008 y el primer trimestre del 2009. Esta investigación tenía como objetivo generar una descripción acerca de la estructura valórica de los jóvenes y la articulación de su discurso moral, con la particularidad de que el sujeto de investigación estaba constituido por jóvenes que han sido acogidos en programas residenciales de protección de derecho, es decir, jóvenes que en su infancia han vivido una situación de vulneración de derecho grave por los adultos que tenían la responsabilidad de su cuidado, y que por esta razón el Estado ha encargado su crianza, restitución y promoción de sus derechos a una institución que los ha acogido en un sistema residencial.

La socialización como proceso es fundamental para la internalización de aspectos estructurales referidos al comportamiento social. Junto con esto, entendemos que este proceso de socialización está marcado por lo que Schütz (1962) define como situación biográfica, es decir, por el cúmulo de situaciones y condiciones que a lo largo de la vida del sujeto van marcando su postura en el mundo y sus horizontes de acción en base a la construcción de la realidad social que está cruzada por la forma específica en que cada sujeto entiende el mundo que lo rodea.

Uno de los elementos fundamentales de la socialización primaria, donde se internalizan los referentes sociales, es que debe darse en un ambiente donde el sujeto sea capaz de identificarse con sus interlocutores, es decir, en un grupo de referencia que usualmente es la familia. Junto con esto, el éxito de la socialización se relaciona directamente con el ambiente afectivo en que el niño y niña se desarrolle. En este sentido, si no hay un soporte emocional que apoye el proceso de socialización, la internalización de la estructura social y cultural no será 100\% efectiva. 
Considerando entonces que lo internalizado en el proceso de socialización es fundamental para orientar la acción de los sujetos, es de suponerse que este proceso marca también la forma en que se internaliza cierta estructura valórica y se articula un discurso moral. De esta manera, como se ha planteado, deberíamos suponer que los jóvenes que han pasado por situaciones especialmente complejas durante su infancia poseen diferencias de base en relación a los jóvenes que no han vivido estas experiencias.

Partiendo de este supuesto de base, la investigación que ha dado pie a este artículo tiene como objetivo describir cuál es la configuración de la estructura valórica y del discurso moral en jóvenes que han sido acogidos en sistemas residenciales de protección. Esta situación define un proceso de socialización distinto, fuera del núcleo familiar de origen ("familia biológica") que hipotéticamente afectaría su configuración valórica. De esta manera, la pregunta de investigación que guía este estudio se relaciona con la descripción de dicha estructura valórica y la articulación de un determinado discurso moral. Suponemos a priori, que comparativamente estos jóvenes se constituyen como sujetos moralmente distintos en base a las peculiaridades de su historia de vida, en este sentido, lo que se busca secundariamente es describir las discrepancias y similitudes que en el discurso surgen entre ambos sujetos jóvenes.

Los resultados obtenidos en la investigación dan cuenta de la postura moral de los jóvenes, en cuanto refleja la forma en que ellos estructuran su discurso sobre cuestiones relativas a su disposición frente a situaciones cotidianas y temas atingentes a lo valórico-moral. En el presente artículo se describirán los principales resultados de la investigación "Discurso moral y estructura valórica en jóvenes acogidos en programas residenciales de protección", concluyendo con un perfil moral de los jóvenes, es decir, con una tipificación ideal del sujeto que ha participado en esta investigación. 
La articulación del discurso moral y la estructura valórica en jóvenes acogidos en residencias de protección de derecho: Perfil de sujeto moral

\section{Aclaraciones preliminares}

Primero, debemos aclarar que la investigación llevada a cabo utilizó dos instrumentos de recolección de datos. Esto dividió el trabajo en terreno en dos etapas, la primera que contempló la aplicación de un cuestionario a 129 jóvenes (un rango menor de la muestra inicial de 180) que corresponde a la totalidad de jóvenes de enseñanza media de los centros estudiados. Luego de eso, se aplicó una entrevista individual a 12 jóvenes distribuidos en tres zonas del país (norte, centro y sur) pertenecientes a las ciudades de Arica, Antofagasta, Quilpué, Santiago, Concepción y Angol.

Para no cometer errores de inferencia, debemos dejar claro antes de partir que la muestra seleccionada para la aplicación del cuestionario no posee un soporte estadístico, por lo que las conclusiones de esta investigación solo pueden inferirse al mismo grupo. La malla de cuotas propuesta solo la componen casi la totalidad de los jóvenes pertenecientes a una institución de cuidado infantil, por lo que no se puede aplicar a todos los jóvenes acogidos en estas residencias ni a todos los jóvenes en general.

Para la recolección de datos cuantitativa, se utilizó una técnica de muestreo por cuotas, definidas por ciertos criterios teóricos de acuerdo a la intencionalidad que esta investigación persigue en la recolección de datos. En este muestreo, se propuso recoger información de cuestionarios aplicados a 180 jóvenes, de acuerdo a la siguiente matriz de cuotas ${ }^{1}$ :

\begin{tabular}{|c|c|c|c|c|c|}
\hline & Primero medio & Segundo medio & Tercero medio & Cuarto medio & \\
\hline ZN & 15 & 15 & 15 & 15 & 60 \\
\hline ZC & 15 & 15 & 15 & 15 & 60 \\
\hline ZS & 15 & 15 & 15 & 15 & 60 \\
\hline & & & & & 180 \\
\hline
\end{tabular}

Como hemos señalado, las cuotas no pudieron ser llenadas en su totalidad debido a que al momento de ser aplicados los cuestionarios el total de jóvenes que se encontraban dentro del

1 Las zonas definidas son: Zona Norte: Arica, Antofagasta. Zona Centro: Quilpué, Santiago, Curicó, y Zona Sur: Concepción, Angol, Chiloé y Puerto Varas. 
perfil definido era solo de 129. Reiteramos que las inferencias que se pueden hacer con los datos entregados solo se aplican al grupo investigado.

Principales resultados de la investigación: perfil de la estructura valórica y el discurso moral de los jóvenes participantes

A continuación se presentan los principales hallazgos de la investigación realizada, la idea es presentar los resultados de forma de concluir un "perfil moral" de los jóvenes participantes del estudio. Con esto, se revelan las orientaciones valórico-morales que han surgido desde el discurso. Todas las referencias planteadas y cifras entregadas están basadas en los resultados obtenidos del análisis de los datos recolectados.

\section{a) Visualización de la realidad juvenil y de su propio ser}

\section{Caracterización de su ser joven}

Los jóvenes participantes de la investigación dicen sentirse contentos con su vida, se encuentran mayoritariamente satisfechos. Lo que refleja el cuestionario aplicado es un gran nivel de satisfacción con sus vidas, y una ligera diferencia entre hombres y mujeres, que hacen a estas últimas relativamente más satisfechas que los varones.

Además, los jóvenes relacionan la satisfacción personal principalmente con el reconocimiento social de sus acciones y logros. Este reconocimiento se vuelve más satisfactorio si proviene del círculo de seres queridos, familia y amigos. El reconocimiento de los cercanos sobre los logros que los jóvenes atesoran es la razón de sentirse satisfechos. Además, constituye un dato relevante en cuanto a que los jóvenes estudiados han pasado por situaciones especiales en su vida y que a priori podría pensarse que determinan de forma negativa su percepción personal. En este sentido, los jóvenes se sienten altamente satisfechos con su vida, lo que siguiendo la relación que ellos mismos describen, hace pensar que sus acciones y logros son reconocidos socialmente. 
La articulación del discurso moral y la estructura valórica en jóvenes acogidos en residencias de protección de derecho: Perfil de sujeto moral

Las metas logradas que hacen sentir satisfacción a los jóvenes se relacionan principalmente con los logros académicos; el salir del liceo, obtener una certificación técnica o el ingreso a la educación superior es lo que logra el reconocimiento social. Esto relaciona nuevamente la satisfacción con su vida con las expectativas de éxito en las metas propuestas. La relación casi lineal que los jóvenes describen vincula la satisfacción -reconocimiento social- éxito personal.

En cuanto al control que los jóvenes tienen de su vida, la mayoría de los jóvenes dice sentirse en control sobre lo que les pasa. Este control está dado por ciertas variables relacionadas. Principalmente son dos los elementos que definen el grado de control que los jóvenes tienen de su vida: a) el nivel de perfeccionamiento y estudios que genera mayores posibilidades de vida independiente en el futuro; y b) la capacidad de opinar, decidir y que las opiniones y decisiones tomadas se respeten y se hagan valer. Esto último es de fundamental importancia, en cuanto a que los programas de acogimiento residencial para niños vulnerados en sus derechos deberían orientar su intervención al enfoque de derechos, donde una de las principales cosas que deberían promover y restituir es el derecho a la participación, y que cada joven que haya sido acogido tenga la posibilidad de construir su proyecto de vida de acuerdo a sus intereses personales.

En cuanto a las diferencias entre hombres y mujeres, son estas últimas las que se sienten ligeramente más en control de su vida que los hombres.

Los jóvenes se describen además como felices. La mayoría de los participantes de esta investigación declara sentirse feliz con su vida, no distinguiéndose esta vez diferencias entre hombres y mujeres. Esta felicidad ha sido descrita como basada principalmente en la familia. La felicidad de los jóvenes está asentada en el cariño y soporte que la familia entrega, el estar estudiando y tener amigos son elementos que también surgen como relevantes. Esto resulta nuevamente muy importante, debido a que los 
jóvenes investigados poseen carencias justamente en este punto. Ellos y ellas se encuentran viviendo temporalmente fuera de su familia de origen, y se encuentran a cargo provisoriamente de una educadora de trato directo que emula el cuidado que una madre podría darle y con un grupo de otros jóvenes, niños y niñas que al igual que él o ella se encuentran en la misma situación. Se esperaba que al vivir las situaciones familiares especiales que han llevado a la vida fuera del hogar de origen, los jóvenes estudiados tuvieran una valoración más compleja de la familia, sin embargo, se ha descubierto que relacionan tanto la felicidad, el éxito y la satisfacción en sus vidas con las relaciones familiares, más aún, han declarado que se sienten felices y satisfechos.

Al momento de pedirles una definición en unas pocas palabras, los jóvenes se han identificado de la siguiente forma:

- Amigables

- Solidarios

- Trabajadores

- Desordenados

- Tranquilos

- Realistas

- Soñadores

- Sociables

- Sinceros

Como puede observarse, los jóvenes mantienen una imagen muy positiva de sí mismos, lo que es coherente con los grados de satisfacción y felicidad declarados. Esto nuevamente tiende a contradecir lo que se ha pensado clásicamente de los jóvenes acogidos en residencias de protección de derechos. Si bien muchas veces se asume que los jóvenes que han vivido esta experiencia en su vida son poco sociables, ellos se definen como amistosos y con facilidad para establecer vínculos sociales.

Respecto de las comparaciones con el mundo adulto, los jóvenes poseen una visión no muy categórica respecto a si son más o menos conservadores que los adultos. Nos encontramos más 
La articulación del discurso moral y la estructura valórica en jóvenes acogidos en residencias de protección de derecho: Perfil de sujeto moral

bien con un conservadurismo moderado en relación al mundo adulto. En este último aspecto, las mujeres tienden a identificarse como ligeramente más conservadoras que los hombres.

Lo mismo ocurre con el individualismo, es decir, los jóvenes en esta investigación no se definen mayoritariamente con los extremos (es decir, más individualista o menos individualista que los adultos), más bien, la mayor parte de las respuestas tienden a concentrarse en un término medio.

Por último, la misma tendencia se define respecto de la tolerancia, los jóvenes no se identifican con extremos sino que con los términos medios en su comparación con los adultos.

Aspectos relevantes en la vida de los jóvenes

Al momento de definir los aspectos más importantes en su vida, los jóvenes mayoritariamente describen que estos son:

- La familia

- Los estudios

- El futuro

Mientras que los menos importantes en su vida son:

- La religión

- La política

Para ellos, la familia posee un especial significado como soporte emocional para la concretización de su proyecto de vida. Si observamos los aspectos más relevantes en la vida de los jóvenes podemos ver la relación que éstos describen:

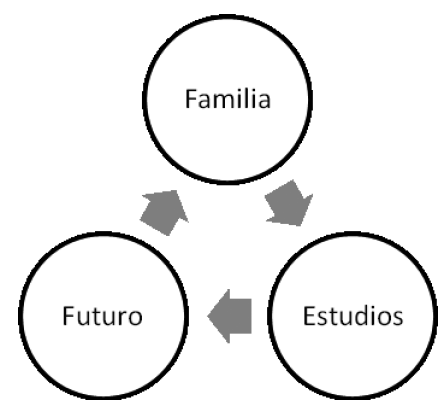


La familia es el soporte necesario para la realización de los proyectos de vida de los jóvenes. Estos proyectos son canalizados mayoritariamente por la capacitación y el desarrollo técnicoprofesional, lo que constituye un elemento de seguridad sobre el éxito futuro. Así mismo, la percepción de satisfacción y el éxito están definidos por la constitución de un nuevo núcleo familiar propio y por la vida independiente.

La religión es uno de los aspectos que con menor frecuencia es considerado relevante por los jóvenes. Más bien, y según lo que ha surgido en el discurso de ellos y ellas, la religión tiende a ser relativizada y la creencia en Dios a ser tomada como algo más personal que institucional. Si bien, la mayor parte de los jóvenes dicen pertenecer a un credo religioso, su creencia está más ligada a la forma específica en que ellos la definen, que a la manera que institucionalmente (como la religión lo dice) está determinado. De la misma forma, la política no es entendida como un aspecto relevante en la vida, se percibe un bajo interés en ella y en ejercicio de actividades propias de la ciudadanía, como votar o dar opiniones respecto de la realidad política nacional. Más bien, los jóvenes entienden la política como la institucionalidad social que mantiene el statu quo y el orden a nivel estructural, es decir, reconocen la importancia de la institucionalidad política para dirigir y llevar al país con cierto rumbo, pero muestran poco interés por ser partícipes de dicha institucionalidad, más bien (al igual que con la religión), más que participantes de estos aspectos son observadores de lo que sucede.

\section{b) Asignación de importancia a valores}

Los jóvenes en su discurso y en lo que han descrito como su estructura valórica, definieron ciertas orientaciones de valor que describen la importancia de determinados aspectos sobre otros. El primero de ellos es la jerarquización de características que buscan en una persona al momento de considerarla amiga o amigo. Además de esto se les ha pedido que escojan una serie de valores según su importancia. 
La articulación del discurso moral y la estructura valórica en jóvenes acogidos en residencias de protección de derecho: Perfil de sujeto moral

\section{Valores en consideración para escoger amigos}

Tomando en cuenta las respuestas entregadas por los jóvenes investigados, podemos concluir que en la situación de la elección de las amistades, un amigo o amiga debería poseer al menos las siguientes características:

- Ser respetuoso

- Honesto

- Leal

- Sincero

- Buen compañero

- Comprometido

Hay pocas diferencias entro hombres y mujeres, pero en lo medular, los hombres buscan más el respeto, compañerismo, bondad y lealtad, mientras que para las mujeres, lo principal es que los amigos sean honestos, leales y sinceros. Si bien lo que se ha descrito al principio es la distribución general de respuestas, se pueden apreciar diferencias entre ambos grupos.

Junto con esto, las características menos valoradas son coherencia, perseverancia y religión (aspecto repetitivo en su baja valoración).

Teniendo en consideración lo que los jóvenes han definido en los discursos, lo que buscan en un amigo es principalmente sinceridad y lealtad. El amigo o amiga es la persona en la que los jóvenes depositan su confianza y de la que esperan lealtad. Hay en este sentido una diferenciación entre las relaciones de amistad, compañerismo y pareja. Las primeras están definidas por lo que hemos descrito anteriormente, las relaciones de compañeros son más bien superficiales y buscan solo la compañía de un par en determinadas situaciones. Las relaciones de pareja son descritas por los jóvenes en un grado más alto de confianza y están definidas por el compromiso que busca el acompañamiento en la realización de proyectos de vida. La diferencia principal tiene que ver con los grados de involucramiento en la vida de los jóve- 
nes, si bien se reconoce la relación de amistad como un vínculo duradero, que posiblemente prevalezca toda la vida, la relación de pareja constituye un grado de compromiso mayor, que si bien puede ser relativizado en su duración, no puede serlo en su nivel de lealtad y fidelidad.

Importancia de algunos aspectos en la vida de los jóvenes

En este punto, los jóvenes una vez más se describen pero en relación a la importancia que algunos aspectos tienen en su vida.

En resumidas cuentas, los jóvenes que participaron en esta investigación plantean los siguientes aspectos como los más importantes en su vida:

- Desarrollarse como persona

- Ser tolerante y respetuoso con los demás

- Tener buenos modales

- Ser honrado y leal

- Ser solidario y

- Tener capacidad para disfrutar

Nuevamente, en los valores descritos con menor relevancia se encuentra la fe religiosa. Diferenciadamente, los aspectos más representativos para los hombres participantes son: desarrollarse como persona, tolerancia y respeto hacia los demás y capacidad para disfrutar. Esto es relativamente coherente con lo que sin hacer diferencias entre grupos se había obtenido como resultado general. Diferente es el caso del grupo de las niñas, donde los conceptos que más se repitieron fueron: tolerancia y respeto hacia los demás, honradez y lealtad y espiritu de superación.

c) Aplicación de valores a situaciones

\section{Valores aplicados a situaciones}

Enfrentados los jóvenes a una serie de situaciones, estos han declarado las siguientes opiniones: 
La articulación del discurso moral y la estructura valórica en jóvenes acogidos en residencias de protección de derecho: Perfil de sujeto moral

a. Copiar en pruebas: Se muestran mayoritariamente en contra de esto, lo que resulta coherente con la alta valoración que poseen a la honradez. Sin embargo, dos de cada diez de los participantes dicen estar de acuerdo.

b. Quedarse con cosas prestadas: casi la totalidad de los jóvenes se encuentra en desacuerdo con esto.

c. Consumo ocasional de marihuana: mayoritariamente los jóvenes se encuentran en desacuerdo con esto (cerca de seis de cada diez). No es menor en todo caso que dos de cada diez están de acuerdo.

d. Consumo ocasional de alcohol: La mitad de los jóvenes está en desacuerdo con esto, sin embargo a casi un cuarto de los participantes le es indiferente. Entre hombres y mujeres se diferencian en cuanto los primeros parecen ser levemente más tolerantes con el tema.

e. Mentir por interés personal: Mayoritariamente los jóvenes desaprueban este hecho, sin embargo las respuestas afirmativas a esto representan a más de un cuarto de los participantes.

f. Infidelidad en la pareja: Mayoritariamente los jóvenes están en desacuerdo con esto, sin embargo el 30\% de ellos y ellas o es indiferente o lo aprueba.

g. Relaciones sexuales fuera del matrimonio: A la mayoría de los jóvenes (casi el 80\%) le es indiferente o aprueba este hecho.

h. Relaciones homosexuales: Un tercio de los jóvenes desaprueba este hecho, pero la mayoría está de acuerdo.

i. Aborto: Hay una clara desaprobación por este hecho, solo dos de cada diez jóvenes lo aprueba o es indiferente.

j. Divorcio: la gran mayoría de los participantes dice estar de acuerdo con que las parejas puedan regularizar sus separaciones.

k. Eutanasia: la opinión en este punto es un poco más dividida, en cuanto seis de cada diez jóvenes dice estar de acuerdo o le es indiferente. Los otros cuatro están en desacuerdo. Entre hombres y mujeres, estas últimas tienen una tendencia más marcada a la desaprobación. 
1. Suicidio: La mayoría de los jóvenes es indiferente o derechamente lo desaprueba (seis de cada diez no está de acuerdo). Las mujeres marcan más notoriamente que los hombres su desaprobación por este hecho.

m. Relaciones sexuales ocasionales con desconocidos: Las respuestas sobre esto se ven dispersas pero con una tendencia hacia la desaprobación (cerca de cuatro de cada diez jóvenes está en desacuerdo, el mismo número es indiferente al tema, el resto lo aprueba).

\section{Aplicación de valores frente a la organización político- social}

Para esta parte de la investigación se les ha pedido a los jóvenes que opinen sobre algunos aspectos referidos a la organización social y la forma en que políticamente se organiza el país.

Sobre esto, los jóvenes se inclinan mayoritariamente a que el Estado debe asegurar la satisfacción de necesidades básicas, es decir, debe intervenir por hacer que ciertos derechos sociales se cumplan. Respecto a la distribución del ingreso, las respuestas son menos categóricas aunque los jóvenes se inclinan con una mayoría relativa hacia el hecho de que los salarios de las personas deberían guardar menos distancia. Casi la totalidad de los jóvenes opina que quien tenga información relevante para hacer justicia frente a un caso, debe entregarla para facilitar el proceso. Finalmente, lo jóvenes dicen que el respeto al medio ambiente es más importante que el desarrollo económico.

\section{Discusión sobre el bien y el mal}

Consultados sobre la objetividad o subjetividad de lo bueno y lo malo, es decir, de la dependencia en las circunstancias de los juicios morales sobre las acciones, no hay una respuesta categórica. No se puede concluir una respuesta categórica en este punto, es decir, los jóvenes tienden a distribuirse parejamente entre las opciones de que independientemente de las circunstancias siempre se sabe lo que es bueno y malo. 
La articulación del discurso moral y la estructura valórica en jóvenes acogidos en residencias de protección de derecho: Perfil de sujeto moral

\section{Igualdad y libertad}

Consultados por la importancia de la libertad y la igualdad, ocurre una situación relativamente similar que en la consulta sobre el bien y el mal. Si bien esta vez sí existe una mayoría estadística que concentra sus respuestas en torno a que la igualdad es más importante que la libertad, esta diferencia no es significativa. Solo seis de cada diez jóvenes se inclinan por la importancia de la igualdad versus la libertad, mientras que los otros cuatro consideran la libertad como un valor más relevante que la igualdad.

\section{d) Sentido de pertenencia y compromiso social}

\section{Identificación y orgullo con el país}

Ante el grado de identificación que los jóvenes tienen con su país, la mayoría dice estarlo, sin embargo, cerca de cuatro de cada diez jóvenes le es indiferente o sencillamente no se identifica con el país. Esto se hace más patente en las entrevistas, donde los jóvenes no muestran una clara tendencia a la identificación. Se percibe en este sentido, más que pertenencia e identificación, un arraigo por ser el país donde nacieron, pero no una identificación consciente y decidida en términos de algún aspecto determinado.

Ocurre los mismo con el orgullo, si bien la mayoría estadística de los jóvenes dice sentirse orgulloso del país, persiste un buen número de participantes que les es al menos indiferente el tema.

\section{Compromiso con otras personas}

Junto con lo anterior se les solicitó a los jóvenes que, de una lista de grupos sociales, señalaran el compromiso que sienten con sus condiciones de vida. En el cuestionario aplicado, los grupos se ordenaban desde grupos de pertenencia más cercanos a grupos más globales y que tienen una relación menos personal con los jóvenes. 
Frente a estos datos, se puede concluir lo siguiente:

a. Como es de esperarse, el nivel de compromiso aumenta a medida que el grupo aludido es más cercano al joven. Esto queda claro cuando vemos que el porcentaje de compromiso más alto lo tiene la familia y el más bajo los latinoamericanos que como grupo social posee una referencia más lejana a lo cotidiano.

b. Fuera de esta consideración inicial, la humanidad (la categoría más amplia del espectro de posibilidades) concentra un alto grado de compromiso.

c. Además, podemos ver que el segundo grupo con más alto sentimiento de compromiso son los compañeros de estudio, donde siete de cada diez jóvenes dicen sentirse comprometidos.

d. Luego de esto, se observa que las categorías personas del vecindario, personas de mi región, personas del país y latinoamericanos concentran los mayores puntajes en la indiferencia.

\section{Opciones y adhesión religiosa}

Describiendo a los jóvenes participantes de esta investigación, se puede decir que en su mayoría son jóvenes creyentes, es decir, se identifican como pertenecientes a una religión. Junto con esto, mayoritariamente son católicos o evangélicos (estos últimos muchos menos que los católicos), donde diferenciados por sexo, son las mujeres las que se identifican como más creyentes y porcentualmente son más las mujeres pertenecientes a la iglesia evangélica.

La adhesión religiosa posee un elemento peculiar pero esperado en cuanto se consulta por la frecuencia en la participación en ciertas ceremonias. Si bien la gran mayoría de los participantes tener una religión y esta es la católica, una buena parte de los participantes afirma no asistir con regularidad (prácticamente nunca) a una ceremonia religiosa. Cerca de cuatro de cada diez jóvenes afirman asistir nunca o prácticamente nunca a un rito 
La articulación del discurso moral y la estructura valórica en jóvenes acogidos en residencias de protección de derecho: Perfil de sujeto moral

religioso fuera de los matrimonios, bautizos y otras ceremonias especiales. Junto con esto, dos de cada diez jóvenes afirma solo asistir en ocasiones especiales, como navidad o semana santa.

Junto con esto, independientemente de su opción por alguna iglesia o credo en particular, los jóvenes en su gran mayoría dicen que Dios es importante en su vida. Tanto así que casi cuatro de cada diez dice que es lo más importante en su vida (esto es más notorio en mujeres que hombres). Esto constituye un elemento importante para el análisis, en cuanto esta cifra es coherente con la cantidad de participantes que declara ser creyente pero demuestra un cierto desapego con la institucionalidad religiosa en cuanto declaran una asistencia muy esporádica a ritos y ceremonias religiosas. Esta hipótesis se vio corroborada por las entrevistas realizadas.

\section{Opciones políticas}

Antes de las conclusiones de este punto, debemos recordar que los aspectos relacionados con la política son muy bajamente valorados por los jóvenes participantes. En este sentido, es de esperarse que haya una cierta cantidad de ellos que no entiendan o comprendan totalmente lo que se les plantea.

Una pregunta que pretendió filtrar este hecho era consultar, antes de responder el resto de la batería de preguntas, si se entendía o no el término democracia. Frente a esto la mayoría de los jóvenes dice sí entender. Dividimos así la muestra en dos grupos, el grupo que declara comprender de qué se trata este concepto es el que continúa respondiendo.

Este último grupo de jóvenes declara mayoritariamente que la democracia puede tener problemas pero que sin embargo es el mejor sistema de gobierno que existe. Entre hombres y mujeres, los varones tienden a estar más de acuerdo con esto. Prácticamente la mitad de los participantes no está de acuerdo con la afirmación de que la democracia como sistema genera dificultades en la toma de decisiones; no es menor, en todo caso, que un 
cuarto de los jóvenes no tiene una opinión clara en este punto. Más indiferentes aún se encuentran respecto a la afirmación las democracias no saben mantener el orden, donde casi cuatro de cada diez jóvenes no tiene una opinión categórica en este tema.

En cuanto a la definición de un sector político, mayoritariamente los jóvenes dicen no identificarse con ninguno. En una lectura más fina, hay una leve tendencia, de quienes sí se identifican con un sector, a hacerlo con la izquierda, y lo hacen más claramente los hombres que las mujeres.

\section{Tolerancia social}

Esta parte de la investigación se relaciona con las opiniones de los jóvenes sobre otros grupos sociales y étnicos, así como la confianza que ellos depositan en las demás personas.

Frente a una serie de grupos sociales y étnico distintos se les pidió a los jóvenes que definieran cuáles son los grados de tolerancia que tienen frente a ellos. Los grupos mayoritariamente no tolerados son los siguientes:

a) Delincuentes

b) Drogadictos

c) Neo Nazis

d) Alcohólicos

Esto guarda coherencia con los resultados obtenidos en la investigación realizada por el Centro de estudios en Juventud el 2007, en la investigación a la población secundaria juvenil en general.

Los grupos con los que se tiene mayor grado de tolerancia o empatía son:
a. Pobres
b. Familias numerosas
c. Inmigrantes

Estos resultados son muy similares a los obtenidos en la investigación del 2007, junto con la cual podemos compartir las 
La articulación del discurso moral y la estructura valórica en jóvenes acogidos en residencias de protección de derecho: Perfil de sujeto moral

mismas conclusiones. Es claro el hecho de que la menor tolerancia se encuentra determinada por conductas disfuncionales o que representan algún grado de violencia o intolerancia (como los neo nazis), mientras que el menor grado de rechazo se encuentra concentrado en grupos sociales afectados por situaciones de exclusión.

Frente a la consulta sobre la confianza que se deposita en la gente, casi las tres cuartas partes de los participantes declaran que es mejor tener precauciones cuando se da confianza. Nuevamente, estos resultados son muy coincidentes con la investigación realizada el 2007, donde el 77,9\% de los encuestados opta por la misma respuesta. De la misma forma, coinciden la segunda y la tercera frecuencia:

- Es mejor no confiar en la gente (2009: 17,8\%; 2007: 14,4\%).

- Se puede confiar en la mayoría de la gente (2009: 9,3\%; 2007: $7,7 \%)$.

En cuanto a la tolerancia con los inmigrantes que vienen a trabajar a nuestro país, la mayor parte de los jóvenes dicen que deben hacerlo en la medida que haya trabajo disponible. Por otro lado, una cuarta parte de los participantes indica que se debería dejar que vengan a trabajar a nuestro país todas las personas que lo deseen), y casi la misma proporción de jóvenes dice que se deberían poner límites estrictos a los extranjeros para que puedan venir a trabajar acá. Un grupo muy menor de los participantes indica que se debería prohibir a las personas de otros países venir a trabajar acá.

Frente a la totalidad de respuestas en torno al tema de los inmigrantes, las opiniones de los jóvenes pueden ser agrupadas de la siguiente forma:

- Las opiniones favorables a que los extranjeros vengan a trabajar en nuestro país concentran la mayoría de las respuestas, es decir, el $69 \%$ de los jóvenes cree que de alguna forma los extranjeros deben poder trabajar en nuestro país.

- Las opiniones desfavorables a que los extranjeros vengan a trabajar 
en nuestro país concentran la minoría de las respuestas, es decir, el 31\% de los jóvenes está en contra de que los inmigrantes trabajen en Chile.

\section{e) Valoración sobre cambios en el futuro}

Para esta parte de la investigación, se les ha pedido a los jóvenes que se refieran al futuro, y sobre todo a sus percepciones y valoraciones sobre ciertos cambios. Concretamente se les pidió a los jóvenes que hicieran una valoración sobre ocho posibles cambios sociales en el futuro. Frente a esto se puede concluir lo siguiente:

Importancia del dinero: la mayoría de los jóvenes valora positivamente el hecho de restar importancia al dinero y los bienes materiales. Enfrentadas las opciones negativas con las positivas sobre este tema, vemos que prácticamente seis de cada diez de los participantes opinan que sería por lo menos bueno que este cambio se generara, mientras que cerca de dos de cada diez opinan que sería malo. No es menor, en todo caso, considerar que también dos de cada diez jóvenes dicen ser indiferentes sobre este tema.

- Que el trabajo tome un lugar menos importante en nuestras vidas: En este caso, se observa una distribución más o menos pareja para las respuestas positivas y negativas. Frente al tema la frecuencia de respuestas positivas a esto comprenden casi cuatro de cada diez participantes, mientras que las negativas concentran prácticamente el mismo puntaje. Se observa una leve superioridad de las respuestas negativas pero no superan los 4 puntos porcentuales. El puntaje obtenido para la respuesta me da lo mismo supera levemente el $17 \%$.

- Que se desarrollen nuevas tecnologías: Como era de esperarse, la gran mayoría de los participantes dice que sería un cambio positivo.

- Que se respete más a la autoridad: las respuestas fueron mayoritariamente favorables, siete de cada diez jóvenes lo con- 
La articulación del discurso moral y la estructura valórica en jóvenes acogidos en residencias de protección de derecho: Perfil de sujeto moral

sideran bueno. Esto es coherente con lo que se ha señalado para otras preguntas y variables en cuanto los participantes tienden a mostrar cierto rechazo a conductas anómicas o claramente antisociales (como intolerancia a la delincuencia, alcoholismo, drogadicción, etc.). Las respuestas desfavorables representan un muy bajo porcentaje del total.

- Mayor valor de la familia: Como es coherente con el resto de los resultados obtenidos, la gran mayoría (nueve de cada diez jóvenes) considera este cambio futuro como muy favorable. Un número insignificante de jóvenes lo considera negativo.

- Que se valore más vivir de una forma simple y natural: En este caso, ocho de cada diez jóvenes lo considera positivo. Un número menor de ellos lo considera negativo.

- Que se valore el respeto por hacer lo que uno quiere hacer: Aproximando el número de respuestas, nueve de cada diez jóvenes lo consideran positivo, mientras solo a uno de cada diez le es indiferente.

- Valoración y respeto por la diversidad sexual: En este último punto, seis de cada diez jóvenes lo consideran positivo, solo uno lo considera negativo y a tres les es indiferente.

\section{f) Asociaciones al éxito personal}

En esta parte final de la investigación se enfrenta a los jóvenes a determinadas situaciones que en su vida cotidiana los hacen tomar decisiones en su acción, las que son orientadas por valoraciones que definen su opinión respecto al éxito de las acciones o situaciones en su vida cotidiana.

\section{Éxito en las relaciones de pareja}

Dentro de un conjunto de características que tiene relación con el éxito de las relaciones de pareja, se les pidió a los jóvenes que las jerarquizaran, obteniéndose el siguiente orden²:

2 Debemos aclarar que como criterio para considerar estos puntajes como los más importantes se han seleccionado los que superan el $80 \%$ de respuestas que lo 
a) Fidelidad

b) Conversar los problemas que se presentan en la pareja

c) Comprensión y tolerancia

d) Respetarse y apreciarse mutuamente

e) Pasar la mayor parte del tiempo juntos

Junto con esto, las valoraciones más bajas son para:

a) Compartir las mismas convicciones religiosas

b) Pertenecer al mismo medio social o clase

c) Estar de acuerdo en cuestiones políticas

Como se ve de forma explícita, los aspectos que se valoran más fuertemente por los jóvenes en una relación de pareja exitosa es que esté basada en la fidelidad, la comunicación, la comprensión, tolerancia y respeto mutuo. Los aspectos poco relevantes se relacionan principalmente con condiciones de clase y las convicciones y opciones religiosas y políticas.

Si diferenciamos a los hombres de las mujeres, tenemos que estas últimas tienden a dar una mayor valoración a los aspectos que aparecen identificados con más relevancia, haciendo especial énfasis en lo que se refiere a comprensión y tolerancia. Si bien las mayorías son las mismas conceptualmente, indistintamente del sexo, las mujeres son más categóricas que los hombres al afirmarlas.

\section{Éxito laboral}

De la misma forma que el éxito en las relaciones de pareja, los jóvenes jerarquizaron los aspectos más relevantes para dar un ordenamiento de las cosas que definen una vida laboral exitosa. Los aspectos descritos de esta forma son:
a) Buen salario
b) Estabilidad en el trabajo 
La articulación del discurso moral y la estructura valórica en jóvenes acogidos en residencias de protección de derecho: Perfil de sujeto moral

c) Buen ambiente laboral

d) Un trabajo en que me guste hacer lo que hago

e) Un trabajo que me permita desarrollarme como persona

f) Que sea un trabajo reconocido socialmente como exitoso

Las características con menor valoración son las siguientes:

a) Que tenga buenas vacaciones

b) Que permita encontrarse o conocer personas nuevas

Ninguna de las condiciones mencionadas anteriormente se ubican en lo poco o nada importante mayoritariamente, de hecho, el criterio para discriminar entre las condiciones más importantes de las menos importantes es que las primeras debían concentrar más del 90\% de las respuestas que las asocien al éxito de manera positiva, mientras que las menos importantes son las que bajan del $80 \%$ de las respuestas. De esta forma, si bien hacemos esta definición de forma conceptual, en la práctica, la totalidad de los aspectos nombrados en el cuestionario fueron considerados como importantes por la mayoría de los participantes.

De esta forma, las condiciones asociadas a una vida laboral exitosa, se resumen en un empleo con buen salario, estabilidad, buen ambiente laboral y la satisfacción personal y social que la labor otorgue. En el análisis diferenciado por sexo no se encontraron resultados significativos.

\section{Condiciones para ser feliz}

En este punto, los jóvenes declaran las siguientes condiciones para ser felices:

a) Desarrollarse como persona

b) Tener una familia

c) Tener buena salud

d) Tener un buen trabajo

e) Tener dinero

De los resultados obtenidos, se puede concluir que los jóvenes asocian su felicidad a condiciones fuera de lo material, y más 
cercanas al éxito personal, esto viene a ser coherente con los resultados obtenidos hasta ahora en otras variables. Junto con esto se puede ver que los aspectos materiales son los que se ubican en último lugar. En este sentido, se puede decir que los jóvenes asocian principalmente su felicidad al desarrollo como persona, la familia y la salud. Diferenciados los hombres de las mujeres, los primeros valoran más ganar dinero y las mujeres dan una mayor valoración a tener un buen trabajo.

\section{Características que debe tener una persona como} modelo a seguir

Los jóvenes participantes definieron, de un conjunto de características, ciertas características que debe tener una persona para convertirse en un ideal a seguir. Estas características describen a una persona de la siguiente manera:
a) Solidario
b) Exitoso personalmente
c) Bondadoso

Las características definidas con menor importancia son:
a) La belleza
b) El dinero
c) El aspecto físico
d) La fama

\section{Asociaciones al éxito en general}

$\mathrm{Al}$ igual que en las variables anteriores, los jóvenes a través de su discurso han descrito una serie de condiciones para definir el éxito personal. Las características definidas son:
a) Ser feliz
b) Vivir de acuerdo a mis propios valores
c) Ser reconocido como profesional
d) Trabajar en lo que a uno le gusta
e) Ser estimado y respetado 
La articulación del discurso moral y la estructura valórica en jóvenes acogidos en residencias de protección de derecho: Perfil de sujeto moral

Las condiciones definidas como menos relevantes son:
a) Tener dinero
b) Tener poder
c) Ser una persona famosa

Entre hombres y mujeres se observan pequeñas diferencias en cuanto a sus tres primeras condiciones prioritarias. Esto se configura de la siguiente forma:

- Orden de las tres primeras prioridades para los hombres: ser feliz $(26,7 \%)$, vivir de acuerdo a mis propios valores $(25,3 \%)$, ser reconocido como buen profesional (17.3\%).

- Orden de las tres primeras prioridades para las mujeres: ser feliz (30\%), ser reconocido como buen profesional (22\%), vivir de acuerdo a mis propios valores (18\%).

A diferencia de los hombres, las mujeres tienen una valoración distinta del éxito profesional; mientras que los varones consideran más relevante vivir bajo sus valores, las mujeres prefieren un reconocimiento en su carrera, lo que resulta coherente con las respuestas entregadas en preguntas anteriores, donde también se le ha dado una mayor valoración al éxito profesional.

\section{Bibliografía}

Baeza, J. (2001). El oficio de ser alumno en jóvenes de liceo de sector popular. Ediciones Universidad Católica Silva Henríquez: Chile.

Baeza, J. (2003). Culturas juveniles, acercamiento bibliográfico. Revista Medellín, $\mathrm{N}^{\circ} 113,7-39$.

Baeza, J. y Sandoval, M., (2007). Configuración de valores en jóvenes estudiantes de la Región Metropolitana. Universidad Católica Silva Henríquez. Inédito.

Berger, P. y Luckmann, T. (1972). La construcción social de la realidad. Amorrortu Editores: Argentina.

Berger, P. (1992). Introducción a la sociología. México: Editorial Limusa.

CERC (1993). Chile: Juventud y Futuro, Una Mirada al Siglo XXI. Santiago: CERC. 
Sandoval, M. (2002). Jóvenes del siglo XXI. Santiago: Ediciones UCSH.

Sandoval, M. (2007). Sub-culturas en la escuela: análisis de sus contenidos y relaciones, en: Revista Temas Sociológicos. Ediciones UCSH.

Schütz, A. (1962). El problema de la realidad social. Argentina: Amorrortu Editores.

Schütz, A. (1964). Estudios sobre teoría social. Argentina: Amorrortu Editores.

Schütz, A. (1993). La construcción significativa del mundo social. Introducción a la sociología comprensiva. Paidós: Argentina.

PNUD, INJUV, Transformaciones culturales e identidad juvenil en Chile.

www.risalc.cl:9090/archivos_recursos/396/10_Biblioteca_pnud_injuv.pdf

INJUV (2005). Prácticas y estilos de vida de los jóvenes del siglo XXI. www. injuv.gob.cl

MIDEPLAN (2000). Los jóvenes chilenos; cambios culturales, perspectivas para el siglo XXI. www.mideplan.cl/admin/docdescargas/ centrodoc/centrodoc_199.pdf 